\title{
Employing beam-forming for estimating the direction of arrival in a multi-path propagation environment
}

\author{
M. Bresch ${ }^{1,2}$, J. Shi ${ }^{1}$, and R. Kokozinski ${ }^{1}$ \\ ${ }^{1}$ Fraunhofer-Institut für Mikroelektronische Schaltungen \& Systeme, Finkenstraße 61, 47057 Duisburg, Germany \\ ${ }^{2}$ now at: Infineon Technologies Austria AG, Siemensstrasse 2, A-9500 Villach, Austria
}

\begin{abstract}
Due to recent researches on traffic accidents with vulnerable road users (VRUs), several measures revealed a great opportunity of reduction. However, all measures applied so far failed to reduce the number of traffic accidents if there is no line-of-sight. Therefore, a transponder signal is utilized to make the VRU visible. The motor vehicle carries a mobile receiver for VRU detection and location. The receiver employs digital beam-forming for estimating the direction of arrival (DOA) with an antenna array for RF ISM band. A sequence of DOA estimations is used for location and motion estimation purposes.
\end{abstract}

\section{Introduction}

In the recent couple of years, the number of traffic casualties has been vastly reduced by several methods. However, none of all methods applied so far has an impact on the traffic accidents if there is no line-of-sight between a driver of a motor vehicle and a vulnerable road user. Therefore, a transponder is utilized to make the road user visible. Thus, traffic accidents may be avoided or at least mitigated in case of no (or poor) visibility of a VRU, i.e. at night, in foggy environment, rain, mist, or if the VRU is occluded by parking cars, trees, houses, or if the driver just does not look into the direction of the VRU. The VRU carries a transponder, which transmits a unique signature, while a mobile receiver is installed on the motor vehicle. The receiver detects and locates the VRU by receiving his transponder signature in order to warn the driver. In Sect. 2 a system for transponder-based DOA estimation is introduced for which the carrier frequency is determined in Sect. 3. Section 4 deals with the range requirements while Sect. 5 determines the region of interest. Section 6 introduces the warning system, Sect. 7 presents the location and motion estimation procedure, Sect. 8 deals with multi-path propagation, Sect. 9 gives a closer look on the beam-forming system, Sect. 10 introduces the signature and Sect. 11 presents location precision results.

Correspondence to: $\mathrm{M}$. Bresch

(manfred.bresch@infineon.com)

\section{Estimating the direction of arrival}

The mobile receiver locates the VRU of interest by estimating the direction of arrival (DOA) of the received signal. VRUs - mostly pedestrians and bicyclists - can be detected and located even if they are not visible, e.g. when they are occluded by parking cars, or by trees or houses. For DOA estimation, antenna arrays are employed. Such an antenna array achieves a much higher gain than only one single antenna. This leads to a very high angular resolution and precision of DOA estimation. The linear antenna array can be mounted on the car front and consists of vertically oriented dipoles. Mounting on the front can be combined with shading the rear since the front region is much more important. This avoids an angular ambiguity in the direction of arrival. The typical distance between adjacent antenna elements is half the wavelength. However, according to simulations, antenna element insertion in between to a distance of one eighth of the wavelength while maintaining the overall array size and increasing the number of elements by a factor of four significantly enlarges the gain and the angular precision. Since the distance between adjacent antenna elements is important for the angular precision, mechanical oscillations of the antenna array would be disturbing. However, typical amplitudes of mechanical oscillations of the car body are small enough so that these do not interfere the quality of DOA estimation. A circular antenna array would lead to a constant angular resolution and precision at any angle, but first of all, this cannot be suitably mounted on the car and, moreover, achieves less gain and angular resolution. A 2-D positioning is sufficient, since the VRU can be assumed to move on the ground. Therefore, azimuthal determination suffices and elevation determination is dispensable. For positioning purposes, a TOA (time-ofarrival) estimation as used e.g. in the global positioning system is too imprecise by far, since it would require analog-todigital converters with a sampling rate of more than $100 \mathrm{MHz}$ to achieve three meters precision. The determination of the RSS (received signal strength) is not feasible as well, since the VRU shall be located behind attenuating obstacles while recent developments of positioning systems based on RSS estimation (which feature high precision) can only be employed for VRU location purpose, if there is no occluding 
obstacle. Utilizing a radar detector would inconveniently require the VRU to wear some metallic burden. Moreover, a VRU cannot be located behind a metallic obstacle.

\section{Carrier frequency determination}

For the determination of the carrier frequency, only ISM bands are eligible. The carrier frequency must not be too high, since then the attenuation by occluding objects is too high and an occluded VRU is not visible. On the other hand, the carrier frequency shall not to be too low either, since in this case, the antenna dimensions are too large to fit on the car front. Therefore, $868 / 915 \mathrm{MHz}$ or $2.45 \mathrm{GHz}$ are appropriate carrier frequencies for the data transmission. Measurements employing a carrier frequency of $2.45 \mathrm{GHz}$ have been carried out. The results show that the transponder signal penetrates obstacles at reasonable intensity. For example, the attenuation by parking cars or trees is less than $15 \mathrm{~dB}$, which is sufficiently low to enable detecting occluded VRUs, since a sufficiently strong signal descending from a line-of-sight propagation, which is necessary for the DOA estimation in a multi-path environment, can be detected by the receiver. At a carrier frequency of $868 / 915 \mathrm{MHz}$, the attenuation is even lower.

\section{Range requirements}

Since the typical speed limit in European cities is $50 \mathrm{kph}$ (about $30 \mathrm{mph}$ ), the maximum vehicle velocity is assumed to be roughly $70 \mathrm{kph}$ since the speed limits are often violated. As the overall stopping distance at this vehicle velocity is about $70 \mathrm{~m}$, the transponder data transmission range has to be about $70 \mathrm{~m}$ as well. Hence, only an active transponder is eligible. A major problem is the implementation of a sufficiently high market penetration of active transponders for the reduction of traffic accidents, since these are heavier than passive transponders. Considerations concerning these issues are still in their early stage. One of the suggestions is utilizing radio controlled clocks. Another proposal is the use of mobile phones as a host for the active transponder. Imaginations also include certain labelled identity cards which, however, will require special batteries, the technology of which is just emerging. Bicyclists' transponders could be implemented in the reflectors.

\section{Region of interest}

The VRU detection systems employing a mobile receiver focuses on the front (the antenna array is mounted on the car front and is blind in the rear). The main region of interest is from $15 \mathrm{~m}$ (this is the reaction distance at $70 \mathrm{kph}$ ) to the stopping distance mentioned above. Warning the driver is senseless if the VRU is inside the reaction distance. As pedestrians and bicyclists are most likely to appear about $8 \mathrm{~m}$ or less aside the car, the angle of interest is within $30^{\circ}$ on both sides of the direction of vehicle velocity. Inside the region of interest, the scene to the right of the car is of more importance since pedestrians crossing from the right are mostly invisible whereas pedestrians crossing the street from the left can often be observed accurately by the driver while they are passing the lane for the oncoming traffic. In countries where the traffic must keep to the left it is vice versa.

\section{Three-stage driver warning}

As in an urban environment, the presence of vulnerable road users has to be assumed all the time, a system that reacts on each detected VRU would lead to a constant warning weakening the driver's attention. Therefore, a three-stage warning system shall avoid the constant warning. In the first stage, only a notice "there is a VRU about" giving the VRU location shall be placed on the screen. In the second stage, the driver receives a warning, that an accident is likely to happen. In the third stage, the driver is strongly encouraged to react, since otherwise an accident cannot be avoided. The third stage could be combined with a pre-crash system.

\section{Positioning and motion detection}

As the vehicle moves on, a sequence of multiple DOA estimations is utilized to spot the VRU location. This kind of sequential triangulation can be used to locate the VRU. The VRU is assumed to move much slower than the vehicle. A precise location can be used for motion estimation, which can be help to reduce traffic accidents, since a VRU crossing the street causes more danger of accident than a VRU moving along the street.

\section{Multi-path propagation}

A very important problem concerning the location by DOA estimation is the multi-path propagation. A discrimination between a line-of-sight propagation and reflection is inevitable in typical urban environment, since a lot of reflections are highly probable. For this discrimination, the sequence of angles-of-arrival of all received signals is analysed. For example, if a certain signal is received from the same direction all the time, it is very likely to be a reflection from the vehicle carrying the receiver or maybe from the car in front, so it is not a signal of interest. Such reflection can be suppressed by means of signal processing. A directly propagated wave moves in a characteristic way as the car moves on. This information can be exploited for echo discrimination. The typical motion of the line-of-sight propagation is stored in the receiver's data base and is compared with all received signals. A time sequence of DOA estimations is used to generate one angle-in-time function for each received signal. The generated angle-in-time functions are analysed by means of supervised pattern recognition. During a receiver's training phase, a car moves passing a transmitter and estimates 


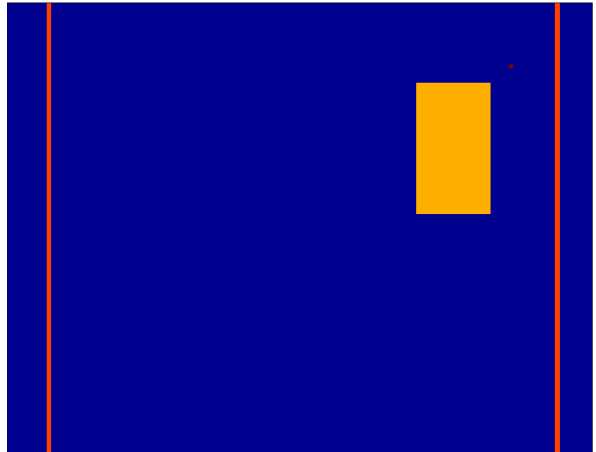

Fig. 1. Standard simulation scenario.

the DOA sequence. A feature vector is extracted from the generated angle-in-time function and stored in the receiver's data base. From each angle-in-time function of the received signals, feature vectors are extracted in the same manner as the stored vector and compared with the vector stored in the data base. As classifier, a maximum-likelihood classification can be used. Another approach is a (symmetric) phase-only matched filtering, which performs a correlation of the phases of two signals to be compared. For the feature extraction, a multi-channel filtering can be used, where the power of each channel output results in one feature. In case of feature extraction using multi-channel filtering, Gabor filters are well suited, since these exhibit optimal time-frequency resolution product. In this case, (symmetric) phase-only matched filtering is likely to be the best classification algorithm. This type of phase-correlating filter is very noise robust, which is important, if the feature is not optimally extracted. Furthermore, phase filters exploit the high information content of phases. The feature vector matching best is assumed to be representing the line-of-sight propagation. This sequential DOA analysis fails in case of a stand-still receiver. However, in this case, no VRU detection is necessary, since only a moving vehicle causes the danger of an accident. Furthermore, this analysis fails in case of a VRU which is directly in front of the car, since the DOA is constantly zero degree and cannot be discriminated against a reflection on the car. But a VRU directly in front of the car is very likely to be seen by the driver. During night and in foggy or raining weather conditions, the driver at least receives the notice that a pedestrian is present in the front of the car (although the distance cannot be determined).

\section{Digital beam-forming}

For DOA estimation using antenna arrays, digital beamforming is employed. The received signal is simultaneously analysed by several signal processing channels in parallel, each maximizing the sensitivity and output power from a certain direction. For an angular precision of one degree in a range of thirty degrees on both sides, 61 channels are necessary. Therefore, the receiver structure is an antenna array

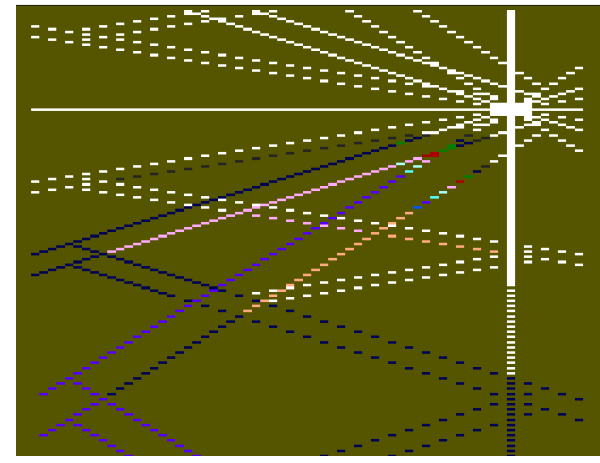

Fig. 2. Multi-path propagation.

connected to an RF front-end, which performs a modulation to an intermediate frequency using a local oscillator. The local oscillator converts the received signal down to a frequency below $10 \mathrm{MHz}$ in order to enable digitisation. This procedure is feasible, since the down-conversion does not influence the phase-differences of the received signal of different antenna elements. The phase-differences of the antenna elements are exploited for DOA estimation. After digitisation, the beam-forming is performed in the digital domain. This is much more robust and less complex than determining the phase relation at RF frequencies. The digital beamforming is just a mathematical procedure and can be carried out for example by a suitable computer program. The received signal of each antenna element is multiplied by a complex factor, that belongs to the channel and to the antenna element. Afterwards all complex products are summed up to achieve the high gain of the antenna array. This results in Eq. (1).

$s_{E}(x, y, t)=\sum_{i=0}^{I} \widehat{s}(i) e^{j\left(k_{x i} x+k_{y i} y-\omega t\right)}$,

where $i$ represents the $\mathrm{i}$-th channel. $I$ is the number of channels, while $k_{x i}$ and $k_{y i}$ are the wave propagation constants along and across the street, respectively, and $\omega$ is the angular frequency of the incident wave. The incident waves of channel $i$ are marked with $\widehat{s}(i)$ and the summed up signal is $s_{E}$. This procedure is carried out in each channel in parallel, and the received signal is interpreted as arriving from the direction of the referring channel, which produces the largest output. Simulations show, that arrival of several signals from various directions does not result in a broad smeared peak, but in several sharp peaks, so that a multi-path discrimination as echo cancellation to determine the line-of-sight arrival is feasible. Figure 1 shows a standard simulation scenario with a road and reflecting sidings, a VRU as transponder signal source and an attenuating car. Figure 2 shows a simulation of multi-path propagation. 


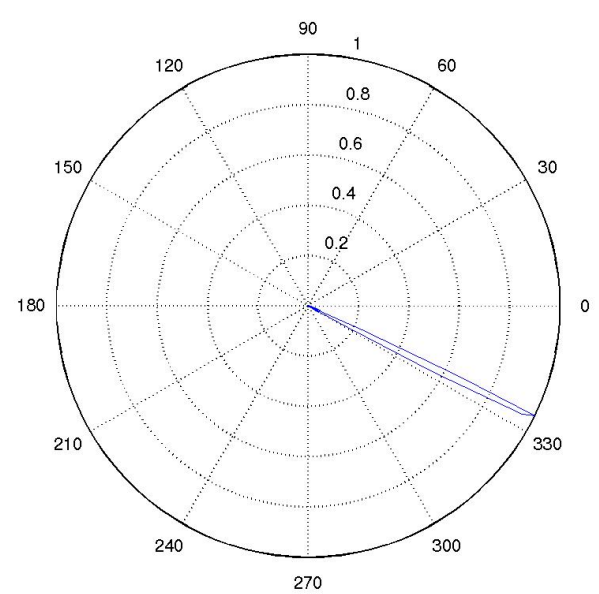

Fig. 3. Directional pattern using 33 antennas at $2.45 \mathrm{GHz}$ (linear).

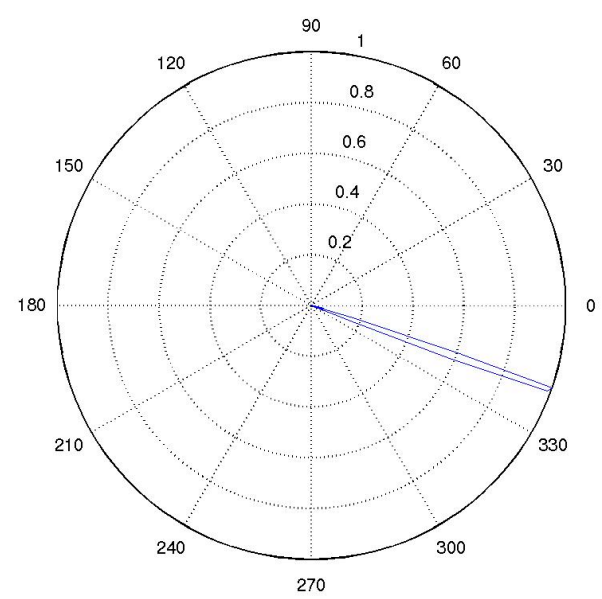

Fig. 4. Directional pattern using 33 antennas at $2.45 \mathrm{GHz}$ (linear).

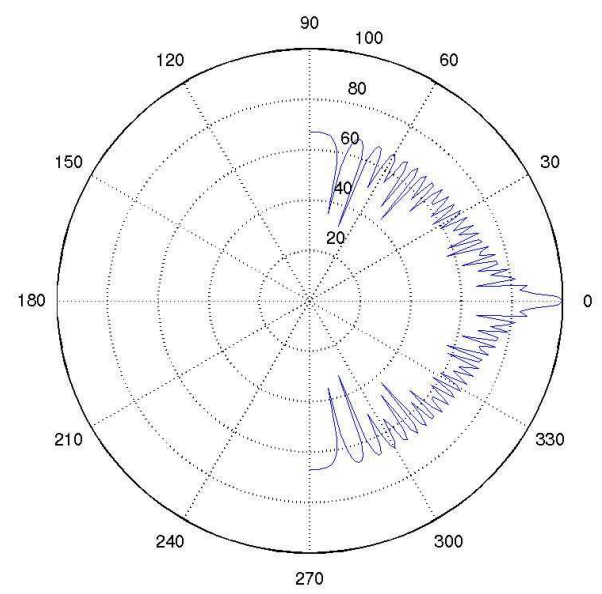

Fig. 5. Directional pattern using 45 antennas at $868 / 915 \mathrm{MHz}$ (in $\mathrm{dB})$.

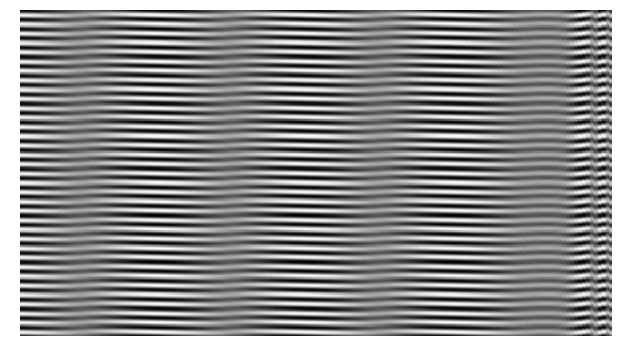

Fig. 6. Simulating three incident waves.

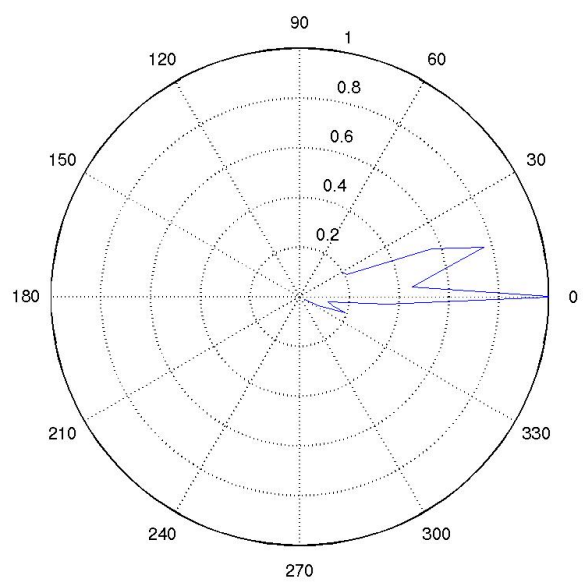

Fig. 7. Simulating received signal strength depending on DOA. Three distinct peaks can be observed.

\section{Unique signature}

In case of the presence of several vulnerable road-users which is always probable in urban environments, a discrimination between the signals of different transponders is necessary. A world-wide unique signature of at least 32 bit serves for this purpose. This signature can moreover be used

to distinguish between a VRU and an interferer (e.g. descending from a bluetooth-signal, another RFID-system, or from a microwave stove). This avoids the near-far problem. The signature can provide important information about the VRU for accident avoidance, e.g. a blind person, a child, a disabled person, bicyclist, bicycle properties and so on.

\section{Location precision}

If the distance between adjacent antenna elements is half the wavelength, this is about $17 \mathrm{~cm}$ at a carrier frequency of $868 / 915 \mathrm{MHz}$ and about $6 \mathrm{~cm}$ at a carrier frequency of $2.45 \mathrm{GHz}$. Therefore 11 elements at $868 / 915 \mathrm{MHz}$ and $29 \mathrm{el}-$ ements at $2.45 \mathrm{GHz}$ fit to a $1.7 \mathrm{~m}$ wide car front. As to be seen from Figs. 3 and 4, simulation results show an angular resolution of 3 deg employing 33 antennas at $868 / 915 \mathrm{MHz}$. The results are shown for two parallel channels maximizing the 
received signal strength from two different angles. The resolution at $2.45 \mathrm{GHz}$ is higher. Simulation results based on further antenna element insertion to 45 antennas equally spaced at one eighth of the wavelength (about $4.2 \mathrm{~cm}$ ) result in an angular resolution of $1 \mathrm{deg}$. Figure 5 shows the simulation results employing 45 antennas at spacing of one eighth wavelength. At $2.45 \mathrm{GHz}$, the resolution is less (i.e. better) than one degree. Employing sequential triangulation for VRU location, this leads to a precision in the cross direction of $1.2 \mathrm{~m}$ in the worst case of $70 \mathrm{~m}$ distance and best case of $0 \mathrm{deg}$ DOA. The precision in the direction of vehicle velocity, the precision depends on the time interval between two successive DOA estimations. In case of a car velocity of $70 \mathrm{kph}$ and a time interval of $200 \mathrm{~ms}$ between two consecutive measurements, the longitudinal precision is about $2.5 \mathrm{~m}$, if the VRU is $70 \mathrm{~m}$ in front of and $15 \mathrm{~m}$ aside the receiver. The precision increases with decreasing distance and decreases with decreasing angle with respect to the vehicle velocity. This is a much higher precision than can be obtained from a TOA based positioning system. Figures 6 and 7 give evidence, that three incident waves lead to three sharp peaks and not to a smeared one.

\section{Conclusion}

We have presented a novel location system based on DOA estimation employing digital beam-forming. The location algorithm is suitable for detecting occluded objects at a precision of about $2 \mathrm{~m}$ and utilizes transponder signals in the $868 / 915 \mathrm{MHz}$ and the $2.45 \mathrm{GHz}$ ISM band. 